\title{
Chemotherapy sensitivity and resistance testing: to be "standard" or to be individualized, that is the question
}

\author{
Tetsuro Kubota ${ }^{1}$ and Larry WeisenthaL ${ }^{2}$ \\ ${ }^{1}$ Center for Advanced and Comprehensive Medicine, Keio University Hospital, 35 Shinanomachi, Shinjuku-ku, Tokyo 160-8582, Japan \\ ${ }^{2}$ Weisenthal Cancer Group, Huntington Beach, CA, USA
}

\begin{abstract}
Radical surgery with extended lymph-node dissection is the treatment of first choice and the only curative treatment for locally advanced gastric cancer. While recent combination chemotherapy with S-1 (a combination of tegafur with two biomodulators, gimeracil and oteracil) has achieved high response rates, controversy still remains regarding the significance of adjuvant cancer chemotherapy after surgery. We have been applying chemosensitivity testing in evaluating the appropriate adjuvant cancer chemotherapy for advanced gastric cancer. Our multiple studies have indicated that this chemosensitivity testing would be useful to improve the results of adjuvant chemotherapy, by increasing survivals in the sensitive group. The chemosensitivity testing is approved as "advanced clinical medicine" by the Japanese Ministry of Health, Welfare, and Labor at 11 institutes at present. While complete lymph-node dissection and chemosensitivity testguided adjuvant chemotherapy has been reported to result in a survival benefit for patients with advanced gastrointestinal cancer, the clinical utility of the testing should be established by means of prospective, randomized clinical trials. Two pivotal clinical trials have been initiated to clarify the utility of chemosensitivity testing in the selection of the appropriate adjuvant cancer chemotherapy for gastric cancer.
\end{abstract}

Key words Chemosensitivity testing - MTT assay · Histoculture drug-response assay · Gastric cancer · Clinical trial

\section{Introduction}

There is no doubt that gastrectomy with extended lymph node resection is the curative treatment of choice for locally advanced gastric cancer. However, because a percentage of patients undergoing curative resection will relapse, adjuvant cancer chemotherapy has been actively studied in gastric cancer. Although many drugs

Offprint requests to: T. Kubota

Received: January 16, 2006 / Accepted: February 8, 2006 have shown activity in recurrent and advanced gastric cancer, the efficacy of single-agent chemotherapy was previously shown to be limited and without survival benefit [1]. The response rates of single-agent therapy were reported to be less than $20 \%$, as shown in Table 1 , with the exception of mitomycin C (MMC), which was tested as a single agent before the present response criteria were introduced. Cisplatin and 5-fluorouracil (5-FU) were each tested on many patients with gastric cancer, and their response rates $(21 \%$ and $19 \%$, respectively) are considered to represent the average efficacy of single agents in gastric cancer. Several combination regimens have been developed, including 5-FU plus adriamycin (ADM) plus MMC (FAM); etoposide plus ADM plus cisplatin (EAP); 5-FU plus leucovorin (LV) plus epirubicin (EPI; FLEP); and 5-FU plus cisplatin (FP). These regimens have achieved $29 \%$ to $48 \%$ response rates, although the overall complete response (CR) rate was only $2 \%$ [2]. Because long-term survival in patients with disseminated malignancy is only achieved when treatments produce CR of the disease, there has been no impact on survival in patients with advanced gastric cancer treated with combination chemotherapy.

Recently, several new drugs have been introduced for gastric cancer, including irinotecan (CPT-11), taxanes (docetaxel and paclitaxel), and S-1 (a combination of tegafur with two biomodulators, gimeracil and oteracil), and this has created a new era of chemotherapy for gastric cancer, with response rates of $44 \%-49 \%$ [3,4]. In particular, combined regimens based on S-1 have been widely studied, combining S-1 with CPT-11, paclitaxel, docetaxel, or cisplatin, with reported response rates of up to $76 \%$ [5] (Table 1). As a result, we currently have a choice between several regimens for advanced gastric cancer, as well as choices in the adjuvant setting. In this article, we review the results of chemosensitivity testing for gastric cancer. In particular, we consider the utility of chemosensitivity testing in evaluating the appropri- 
Table 1. Response rates of antitumor agents and their combinations in advanced gastric carcinoma

\begin{tabular}{llll}
\hline Single agent & \multicolumn{3}{l}{ Combination } \\
\hline Mitomycin C & $30 \%$ & FAM & $29 \%$ \\
Cisplatin & $19 \%$ & FP & $38 \%$ \\
5-Fluorouracil & $21 \%$ & DCF & $39 \%$ \\
Adriamycin & $17 \%$ & ECF & $46 \%$ \\
Docetaxel & $23 \%$ & S-1 + CPT-11 & $50 \%$ \\
Paclitaxel & $25 \%$ & D + cisplatin & $25 \%$ \\
CPT-11 & $23 \%$ & S-1 + D & $46 \%-54 \%$ \\
S-1 & $47 \%$ & S-1 + cisplatin & $76 \%$ \\
\hline
\end{tabular}

F, 5-fluorouracil; A, adriamycin; M, mitomycin C;

E, epirubicin; L, leucovorin; $\mathrm{P}$ and $\mathrm{C}$, cisplatin; $\mathrm{D}$, docetaxel

ate adjuvant cancer chemotherapy for advanced gastric cancer, and we discuss the integration of "standard" and "individualized" therapy in adjuvant cancer chemotherapy for gastric cancer.

\section{Cumulative results of chemosensitivity testing for antitumor agents in Japan}

The Japan Research Society for Appropriate Cancer Chemotherapy set out to summarize the present status of chemosensitivity testing for antitumor agents in Japan [6]. Two different questionnaires were sent to 122 and 94 institutes, respectively, and responses were received from $87(71.3 \%)$ and $41(43 \%)$ institutes, respectively. Results showed that chemosensitivity testing was performed at 42 institutes, where a total of two in vivo and ten in vitro different assay methods were performed. The actual number of cases tested varied from 1 to 368 cases/year per institute, with a median of 15 cases and mean \pm SD of $48 \pm 65$ cases. The total number of tested cases has increased, from 1747 cases in 1993 to 1934 cases in 1994 and to 2147 cases in 1995, resulting in an average of 1891 cases/year. Assays used included the adenosine triphosphate inhibition assay, collagendroplet embedded drug-response assay, fluorescent dye assay, growth-chamber assay, the histoculture drugresponse assay, human tumor clonogenic assay, 3-(4,5dimethylthiazol-2-yl)-2,5-diphenyltetrazolium bromide (MTT) assay (succinic dehydrogenase inhibition [SDI] test), nuclear damage assay, nude mouse model, subrenal capsule assay, and the thymidine incorporation assay (scintillation assay). The correlation of in vitro and in vivo results revealed 215 true-positive (S/S), 246 false-positive (S/R), 45 false-negative (R/S), and 595 true-negative $(\mathrm{R} / \mathrm{R})$ cases, resulting in a $47 \%$ truepositive rate and a $93 \%$ true-negative rate, and $74 \%$ accuracy (Table 2). In other words, patients treated with $\operatorname{drug}(\mathrm{s})$ testing in the "sensitive" range were nearly seven times more likely to respond to chemotherapy
Table 2. Predictive values for chemosensitivity testing

No. of correlations

\begin{tabular}{lccccc} 
attempted & $\mathrm{S} / \mathrm{S}$ & $\mathrm{S} / \mathrm{R}$ & $\mathrm{R} / \mathrm{S}$ & $\mathrm{R} / \mathrm{R}$ & Accuracy \\
\hline 1.101 & 215 & 246 & 45 & 595 & $74 \%$ \\
\hline
\end{tabular}

True positive (S/S), 47\%; true negative (R/R), $93 \%$; sensitivity, $83 \%$; specificity, $71 \%$

$\mathrm{S} / \mathrm{R}$, false positive; $\mathrm{R} / \mathrm{S}$, false negative

than were patients treated with drugs testing in the "resistant" range (47\% versus $7 \%$ ). We concluded that chemosensitivity testing was being widely applied in this country and that it has a high accurate predictive value for advanced carcinomas. Thus, chemosensitivity testing appears to be advantageous for the clinical treatment of several kinds of carcinomas, including the appropriate adjuvant cancer chemotherapy for advanced gastric cancer.

The chemosensitivity testing was approved by the Japanese Ministry of Health, Welfare, and Labor as "advanced clinical medicine", in July 1999, for use at Keio University Hospital, and the number of approved institutes increased to 11 in December 2005 [7].

\section{"Standard" adjuvant cancer chemotherapy for gastric cancer}

Hermans et al. [8], in a metaanalysis, reviewed 14 randomized trials and analyzed data from 11 of these trials, consisting of 2096 patients. When they calculated the odds ratios by comparing the adjuvant-treatment arm with the surgery-alone arm, the results suggested that the adjuvant chemotherapy regimens did not improve survival $(95 \%$ confidence interval for odds ratio of recurrence, 0.78-1.08), in spite of the effectiveness of these regimens in phase II studies. However, Pignon et al. [9] recalculated the odds ratio, of which the upper limit was less than 1.0, in their reappraisal of that metaanalysis of adjvant chemotherapy. Nakajima et al. [10] analyzed ten adjuvant chemotherapy regimens in six clinical protocols, carried out at the Cancer Institute Hospital from 1959 to 1985, consisting of 1185 patients, and suggested that the use of these regimens showed a statistically significant survival benefit in patients who had curative surgery. Following these two metaanalyses, four groups of authors calculated the odds ratios of the effect of adjuvant cancer chemotherapy for gastric cancer, reporting that the upper limits were less than 1.0 in all their metaanalyses [11-14]. However, no one single trial has clarified the usefulness of adjuvant cancer chemotherapy for advanced gastric cancer compared with a surgery-alone arm. Based upon these results, the first edition of the Guideline of gastric cancer therapy of the Japanese Gastric Cancer Association 
states that "Although multiple metaanalyses have indicated prolonged survival with adjuvant cancer chemotherapy, no standard regimen has been confirmed at the present time." [15], and the second edition [16] states: "A randomized control trial with a very large cohort should be carried out with a surgery-alone control arm and survival endpoint. Until that time, "adjuvant cancer chemotherapy is not approved as a clinical practice."

In 2001, Macdonald et al. [17] investigated the effect of surgery plus adjuvant chemoradiotherapy on the survival of patients with resectable adenocarcinoma of the stomach or gastroesophageal junction. They randomized 556 patients with resected adenocarcinoma of the stomach or gastroesophageal junction to surgery plus postoperative chemoradiotherapy or surgery alone. The adjuvant treatment consisted of fluorouracil and leucovorin, followed by $4500 \mathrm{cGy}$ of radiation. The median overall survival in the surgery-only group was 27 months, as compared with 36 months in the chemoradiotherapy group; the hazard ratio for death was $1.35(P=0.005)$ in the surgery-alone arm [17]. As a result, they concluded that adjuvant chemoradiotherapy was indicated for all patients at high risk for recurrence of adenocarcinoma of the stomach or gastroesophageal junction who had undergone curative resection. Although this result was reflected in the guidelines of the National Comprehensive Cancer Network (NCCN) and the Physician Data Query (PDQ) of the National Cancer Institute (NCI), the Japanese Gastric Cancer Association did not recommend this adjuvant therapy as standard, because of the low rate of lymph-node dissection in this study. In another study, by Kinoshita et al. [18], the oral fluoropyrimidine, UFT, was evaluated, in terms of survival benefit, in 93 patients with curatively operated gastric cancer. Patients with T2 and N1-2 advanced gastric cancer were randomized at a 1:1 ratio to a surgeryalone group $(n=95)$ or a surgery plus UFT group who received UFT at $360 \mathrm{mg} / \mathrm{m}^{2}$ per day for 16 months $(n=$ 93). The 4-year overall survival rate was $86.3 \%$ for the UFT group and $73.6 \%$ for the surgery-alone group, with a statistically significant difference, at $P=0.0176$. (Because the planned sample size of this study [18] was not attained, this trial will be re-evaluated in the new Japan Clinical Oncology Group (JCOG) clinical trial including chemosensitivity testing, described below). Cunningham et al. [19] designed the Medical Research Council Adjuvant Gastric Infusional Chemotherapy (MAGIC) trial (ISRCTN 93793971) to determine whether epirubicin, cisplatin, and infused 5-FU (ECF) would have a survival advantage in operable gastric cancer. Five hundred and three patients with operable adenocarcinoma of the stomach, esophagogastric junction, or lower esophagus were randomized to a perioperative chemotherapy group $(n=250)$ or to a surgery-alone group $(n=253)$. The survival hazard ratio (HR) was $0.75(P=0.009)$ and the progression-free survival HR was $0.66(P=0.001)$ for the perioperative chemotherapy group. While this study suggested the value of perioperative chemotherapy, the mortality rate was $6 \%$ in both arms, and the percentage of randomized patients actually started on postoperative chemotherapy was only $55 \%$, while the percentage completing it was only $42 \%$. In a very recent study, S- 1 was employed as adjuvant cancer chemotherapy for patients with curatively operated stage II and stage III gastric cancer and compared with a surgery-alone arm, as the

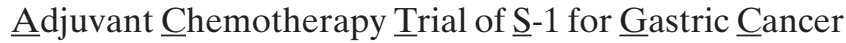
(ACTS-GC); S-1 was administered for 1 year in the chemotherapy arm, with the primary endpoint being overall survival. This study was completed in December 2004 with an accrual of 1059 cases, but we must wait for 3 years to know its result.

\section{Drug sensitivity testing in evaluating appropriate adjuvant cancer chemotherapy for advanced gastric cancer}

\section{MTT assay}

We have introduced chemosensitivity testing to evaluate the appropriate adjuvant cancer chemotherapy for advanced gastric cancer. Our initial study [20] was conducted between July 1988 and October 1992. Patients underwent resection of the primary lesion; a single-cell suspension assay of fresh surgical materials was then tested with an MTT assay, first described by Mosmann [21], with some modifications [22-24]. Fifty patients with pathological stage III or IV gastric cancer were enrolled in this study, of whom 10 received no chemotherapy after the surgery, while 40 received chemotherapy, at similar dose levels, after the surgery. The patients given chemotherapy were retrospectively divided into two groups, consisting of a sensitive group (treated with at least one agent identified as effective by the assay), and a resistant group (treated with agents to which the cells were not sensitive in the assay). The purpose of the study was to identify the optimal cutoff inhibition rate (IR) in the MTT assay for the determination of the appropriate adjuvant cancer chemotherapy after surgery. A cutoff IR of $30 \%$ was optimal for differentiating the survival rates between the sensitive and resistant groups. Patients treated with drugs which showed more than $30 \%$ IR in their surgical specimens showed a better survival rate than patients treated with drugs which were ineffective in the assay. In addition, there were no statistically significant differences between the survival rate of the resistant group and that of the surgery-alone group. 


\section{Histoculture drug-response assay (HDRA)}

Following the above retrospective study, we introduced the HDRA [25-27]. To investigate the potential of the HDRA to contribute to patient survival, 215 patients with gastric cancer, from 45 medical centers, were tested with the HDRA in a blinded study after resection of the primary lesion [28]. All of the registered patients were treated with the same protocol, without knowledge of the results of the HDRA. One hundred and sixty-eight patients received at least $20 \mathrm{mg} / \mathrm{m}^{2} \mathrm{MMC}$ and a minimum of $30 \mathrm{~g} \mathrm{UFT}$, thereby making them eligible for the study. Of these 168 patients, 128 cases were evaluated with the HDRA. The evaluable patient tumors were tested by HDRA, with the $\left[{ }^{3} \mathrm{H}\right]$ thymidine incorporation endpoint measured by microautoradiography defined as drug "sensitive" or "resistant". In the correlation of the overall survival rates of the MMC- and UFT-treated stage III and stage IV gastric cancer patients with the HDRA for MMC, the sensitive group consisted of 25 patients whose tumors were sensitive to MMC on the HDRA. The resistant group consisted of 98 patients whose tumors were resistant to MMC on the HDRA. The overall survival rate of the sensitive group was better than that of the resistant group $(P=0.014$ by logrank test and $P=0.009$ by generalized Wilcoxon test). This difference was also observed in the sensitivity to 5FU. The sensitive group consisted of 20 patients whose tumors were sensitive to 5-FU on the HDRA. The resistant group consisted of 99 patients whose tumors were resistant to 5-FU. The overall survival rate of the sensitive group was better than that of the resistant group $(P$ $=0.031$ by log-rank test and $P=0.028$ by generalized Wilcoxon test). When the sensitivity to both MMC and/ or 5-FU was assessed, the sensitive group consisted of 38 patients whose tumors were sensitive to MMC or 5FU on the HDRA, and the resistant group consisted of 89 patients whose tumors were resistant to MMC and 5FU on the HDRA. In some specimens, only one drug was tested. The overall survival rate of the sensitive group was better than that of the resistant group $(P=$ 0.001 by log-rank test and $P=0.0007$ by generalized Wilcoxon test). Multivariate analysis was carried out according to Cox's proportional hazard model. Risk ratio analysis demonstrated that sensitivity to MMC and/ or 5-FU on the HDRA was an independent risk factor for overall survival in each category. In this blinded study, the overall survival rates of the HDRA-sensitive group were found to be significantly higher than those of the HDRA-resistant group tested. The results demonstrated that the HDRA response was correlated with patient survival, which suggests the potential of use of the HDRA to contribute to survival in gastric cancer patients.

\section{Methods for predicting drug activity in gastric cancer: resistance enzyme testing versus cell-culture ("chemosensitivity") testing}

The two major approaches to predicting the activity of anticancer drugs in gastric cancer have been resistance enzyme testing and cell-culture (hereafter called "chemosensitivity") testing. Resistance enzyme assays are based on measuring the expression of genes coding for specific drug-resistance-related enzymes, measuring the cellular content of these enzymes, and/or measuring the cellular activity of these enzymes. Examples of resistance enzyme assays include thymidylate synthase (TS) $[29,30]$, dihydropyrimidine dehydrogenase (DPD) [3133], and excision repair cross-complementing (ERCC1) [34] gene assays. Increases in TS, DPD, and/or ERCC1 (measured by gene expression, cellular content, and/or cellular enzyme activity) in patients with gastric cancer have been correlated with response to 5-FU (TS and DPD) and to cisplatin (ERCC1), and with survival.

An alternative approach to resistance enzyme testing is cell-culture (chemosensitivity) testing. It should be noted that gene expression is only useful insofar as it predicts enzyme content, and that enzyme content is only useful insofar as it predicts enzyme activity, and enzyme activity is only useful insofar as it predicts drug effects at the cellular level. Chemosensitivity testing is theoretically attractive in that it directly measures net drug effects at the cellular level and does not depend on a clear understanding of all known mechanisms of drug action and mechanisms of cellular resistance to drug action. However, chemosensitivity testing must be performed on fresh, viable tissue, while resistance enzyme assays may be performed on fixed tissues stored in paraffin blocks. In the future, it is likely that both approaches to predicting drug activity will find increasing utility in the treatment of gastric cancer, particularly as technologies (e.g., cDNA microarrays [35]) improve. However, at present, chemosensitivity testing is available for a wider array of drugs and would appear to be the more promising short-term approach toward the individualization of chemotherapy.

\section{Discussion}

In addition to our reports mentioned previously $[20,27,28]$, we have performed several additional studies to compare the survival outcomes of patients with advanced gastric cancer, using the MTT endpoint [36-38]; all the studies indicated that the sensitive patients had a favorable survival outcome (Table 3).

In 2004, Samson et al. [39] reported a systematic review to evaluate evidence, comparing therapy guided by chemotherapy sensitivity and resistance assays with 
Table 3. Usefulness of chemosensitivity testing in evaluating the appropriate adjuvant cancer chemotherapy for advanced gastric cancer

\begin{tabular}{|c|c|c|c|c|}
\hline Stage & Resistant & Sensitive & $P$ value & $\begin{array}{c}\text { Reference } \\
\text { no. }\end{array}$ \\
\hline Stage III/IV & 21 & 19 & $<0.05$ & 20 \\
\hline Stage III/IV & 10 & 22 & $<0.005$ & 27 \\
\hline Stage III/IV & 38 & 89 & $<0.0006$ & 28 \\
\hline Stage III/IV & 13 & 8 & $<0.05$ & 36 \\
\hline Stage III & 11 & 18 & $<0.05$ & 37 \\
\hline Stage III & 51 & 27 & $<0.0001$ & 38 \\
\hline Stage IV (P0) & 42 & 16 & $<0.01$ & 38 \\
\hline
\end{tabular}

empiric chemotherapy, emphasizing survival outcomes. They searched for prospective studies comparing patients treated contemporaneously by assay-guided chemotherapy and by empiric therapy. Their review identified ten studies meeting the selection criteria, plus one retrospective study, using seven different assays. Only two studies randomly assigned patients to assayguided treatment or empiric treatment. One of the two randomized trials found a significantly higher response rate in the assay-guided group. Two nonrandomized studies found overall survival to be significantly improved with assay-guided therapy. Samson et al. [39] concluded that, while higher response rates for assayguided therapy had been observed, the differences may have been attributable to bias or confounding, and little evidence on survival was available. These results do not establish the relative effectiveness of assay-guided treatment and empiric treatment. Randomized trials are needed. Schrag et al. [40] carried out a systematic review, accompanied by a technology assessment of chemotherapy sensitivity and resistance assays, which defined the role of these tests in routine oncology practice. Their literature search did not identify any chemotherapy sensitivity and resistance assays for which the evidence base was sufficient to support use in oncology practice. However, they concluded that, because an in vitro analytic strategy has potential importance, participation in clinical trials evaluating these technologies remains a priority.

The above "systematic review" and "technology assessment" are open to some criticism, as they specifically excluded from consideration all studies (of which there are many [41]) which dealt "only" with the accuracy of the chemosensitivity and resistance assays, while considering only studies (of which there were few, [4143]) relating to the question of whether using the assays in clinical practice actually improved clinical outcomes. This is curious, as all other predictive tests used to choose drug therapy in cancer have been validated only with respect to predictive accuracy and not with respect to whether or not treatment outcomes are improved when therapy is chosen on the basis of information provided by the tests. Examples of commonly used tests which are widely used - even though they have never been shown to improve treatment outcomes - are estrogen receptors, human epidermal growth factor receptor type 2/neu (Her2/neu), and immunohistochemical staining for cell markers. Recently introduced prognostic tests based on gene expression are receiving broad acceptance in the United States, despite the fact that all published studies have shown only retrospective comparisons between test results and treatment outcomes, and none have been shown to improve treatment outcomes [42,43]. Actually, the controversy usually presents as differences between "response" and "survival" as the outcome in evaluating most cancer therapy, including any predictive test. Because the "response" is a surrogate endpoint for "survival", an assay should be validated initially by "accuracy for predicting response" and finally by "survival outcome".

In an attempt to resolve these controversies regarding the use of chemosensitivity testing, two pivotal clinical trials in Japan have just been started, to clarify the usefulness of chemosensitivity testing in evaluating the appropriate adjuvant cancer chemotherapy for gastric cancer. One is a one-arm blinded prospective trial designed by the Japan Clinical Cancer Research Organization (JACCRO) [44], and the other is a randomized control study planned by the JCOG [45].

\section{References}

1. Schipper DL, Wagener DJ. Chemotherapy of gastric cancer. Anticancer Drugs 1996;7:137-49.

2. Macdonald JS, Gohmann JJ. Chemotherapy of advanced gastric cancer: present status, future prospects. Semin Oncol 1988;15:(3 Suppl 4) 42-9.

3. Sakata Y, Ohtsu A, Horikoshi N, Sugimachi K, Mitachi Y, Taguchi T. Late phase II study of novel oral fluoropyrimidine anticancer drug S-1 (1 M tegafur- $0.4 \mathrm{M}$ gimestat- $1 \mathrm{M}$ otastat potassium) in advanced gastric cancer patients. Eur J Cancer 1998;34: 1715-20.

4. Koizumi W, Kurihara M, Nakano S, Hasegawa K. Phase II study of S-1, a novel oral derivative of 5-fluorouracil, in advanced gastric cancer. For the S-1 Cooperative Gastric Cancer Study Group. Oncology. 2000;58:191-7.

5. Koizumi W, Tanabe S, Saigenji K, Ohtsu A, Boku N, Nagashima F, et al. Phase I/II study of S-1 combined with cisplatin in patients with advanced gastric cancer. Br J Cancer 2003;89:2207-12.

6. Kondo $\mathrm{T}$, Kubota $\mathrm{T}$, Tanimura $\mathrm{T}$, Yamaue $\mathrm{H}$, Akiyama $\mathrm{S}$, Maehara Y, et al. Cumulative results of chemosensitivity tests for antitumor agents in Japan. Anticancer Res 2000;20:2389-92.

7. http://www.mhlw.go.jp/topics/0106/tp0601-1a.html

8. Hermans J, Bonenkamp JJ, Boon MC, Bunt AMG, Ohyama S, Sasako M, et al. Adjuvant therapy after curative resection for gastric cancer: meta-analysis of randomized trials. J Clin Oncol 1993;11:1441-7.

9. Pignon JP, Ducreux M, Rougier P. Meta-analysis of adjuvant chemotherapy in gastric cancer: a critical reappraisal. J Clin Oncol 1994:12:877-8. 
10. Nakajima T, Ohta K, Ohyama S, Hamajima N. Meta-analysis of adjuvant chemotherapy trials for gastric cancer at the Cancer Institute Hospital. In: Nakajima T, Yamaguchi T, editors. Multimodality therapy for gastric cancer, Heidelberg Berlin New York Tokyo: Springer-Verlag; 1999. p. 27-31.

11. Earle CC, Maroun JA. Adjuvant chemotherapy after curative resection for gastric cancer in non-Asian patients: revisiting a meta-analysis of randomized trials. Eur J Cancer 1999;35:1059-64.

12. Mari E, Floriani I, Tinazzi A, Buda A, Belfiglio M, Valentini M, et al. Efficacy of adjuvant chemotherapy after curative resection for gastric cancer: a meta-analysis of published randomised trials. A study of the GISCAD (Gruppo Italiano per lo Studio dei Carcinomi dell'Apparato Digerente). Ann Oncol 2000;11:837-43.

13. Janunger KG, Hafstroem L, Nygren P, Glimelius B, SBU-group. Swedish Council of Technology Assessment in Health Care. A systematic overview of chemotherapy effects in gastric cancer. Acta Oncol 2001;40:309-26.

14. Panzini I, Gianni L, Fattori PP, Tassinari D, Imola M, Fabbri P, et al. Adjuvant chemotherapy in gastric cancer: a meta-analysis of randomized trials and a comparison with previous meta-analyses. Tumori 2002;88:21-7.

15. Japanese Gastric Cancer Association, editors. Guideline of gastric cancer therapy. 1st ed. Tokyo: Kanehara; 2001.

16. Japanese Gastric Cancer Association, editors. Guideline of gastric cancer therapy. 2nd ed. Tokyo: Kanehara; 2004.

17. Macdonald JS, Smalley SR, Benedetti J, Hundahl SA, Estes NC, Stemmermann GN, et al. Chemoradiotherapy after surgery compared with surgery alone for adenocarcinoma of the stomach or gastroesophageal junction. N Engl J Med 2001;345:725-30.

18. Kinoshita T, Nakajima T, Ohashi Y, National Surgical Adjuvant Study Group: Adjuvant chemotherapy with uracil-tegafur (UFT) for serosa negative advanced gastric cancer: results of a randomized trial by National Surgical Adjuvant Study of Gastric Cancer. Proc $41^{\text {st }}$ ASCO, Abstract no. 4021; 2005. p. 313s.

19. Cunningham D, Allum WH, Srtenning SP, Weeden $S$ for the NCRI Upper GI Cancer Clinical Studies Group Perioperative chemotherapy in operable gastric and lower oesophageal cancer: final results of a randomised, controlled trial (the MAGIC trial, ISRCTN 93793971). Proc 41 st ASCO Abstract no. 4001; 2005. p. $308 \mathrm{~s}$.

20. Saikawa Y, Kubota T, Furukawa T, Suto A, Watanabe M, Kumai $\mathrm{K}$, et al. Single-cell suspension assay with an MTT end point is useful for evaluating the optimal adjuvant chemotherapy for advanced gastric cancer. Jpn J Cancer Res 1994;85:762-5.

21. Mosmann T. Rapid colorimetric assay for cellular growth and survival: application to proliferation and cytotoxicity assays. J Immunol Methods 1983;65:55-63.

22. Shimoyama Y, Kubota T, Watanabe M, Ishibiki K, Abe O. Predictability of in vivo chemosensitivity by in vitro MTT assay with reference to clonogenic assay. J Surg Oncol 1989;41:12-8.

23. Suto A, Kubota T, Shimoyama Y, Ishibiki K, Abe O. MTT assay with reference to the clinical effect of chemotherapy. J Surg Oncol 1989;42:28-32.

24. Furukawa T, Kubota T, Suto A, Takahara T, Yamaguchi H, Takeuchi T, et al. Clinical usefulness of chemosensitivity testing using the MTT assay. J Surg Oncol 1991;48:188-93.

25. Furukawa T, Kubota T, Watanabe M, Takahara T, Yamaguchi $\mathrm{H}$, Takeuchi $\mathrm{T}$, et al. High in vitro-in vivo correlation of drug response using sponge-gel-supported three-dimensional histoculture and the MTT end point. Int J Cancer 1992;51:489-98.

26. Furukawa T, Kubota T, Watanabe M, Kase S, Takahara T, Yamaguchi $\mathrm{H}$, et al. Chemosensitivity testing of clinical gastrointestinal cancers using histoculture and MTT endpoint. Anticancer Res 1992;12:1377-82.

27. Furukawa T, Kubota T, Hoffman RM. Clinical applications of the histoculture drug response assay. Clin Cancer Res 1995;1:305-11.

28. Kubota T, Sasano N, Abe O, Nakao I, Kawamura E, Saito T, et al. Potential of the histoculture drug response assay to contribute to cancer patient survival. Clin Cancer Res 1995;1:1537-43.
29. Lenz HJ, Leichman CG, Danenberg KD, Danenberg PV, Groshen S, Cohen H, et al. Thymidylate synthase mRNA level in adenocarcinoma of the stomach: a predictor of primary tumor response and overall survival. J Clin Oncol 1996;14:17682.

30. Grau JJ, Domingo-Domenrech J, Morente V, Pera M, GarciaValdecasas JC, Fuster J, et al. Low thymidylate synthase expression in the primary tumor predicts favorable clinical outcome in resected gastric cancer patients treated with adjuvant tegafur. Oncology 2004:66:226-33.

31. Ishikawa $\mathrm{Y}$, Kubota $\mathrm{T}$, Otani $\mathrm{Y}$, Watanabe $\mathrm{M}$, Teramoto $\mathrm{T}$, Kumai K, et al. Dihydropyrimidine dehydrogenase and messenger RNA levels in gastric cancer: possible predictor for sensitivity to 5-fluorouracil. Jpn J Cancer Res 2000;91:105-12.

32. Toriumi F, Kubota T, Saikawa Y, Yoshida M, Otani Y, Watanabe $\mathrm{M}$, et al. Thymidylate synthetase genotype and TS/ dihydropyrimidine dehydrogenase mRNA level as an indicator in determining chemosensitivity to 5-fluorouracil in advanced gastric cancer. Anticancer Res 2004;24:2455-63.

33. Terashima M, Fujiwara H, Takagane A, Abe K, Irinoda $T$, Nakaya T, et al. Prediction of sensitivity to fluoropyrimidines by metabolic and target enzyme activities in gastric cancer. Gastric Cancer 2003;6(Suppl 1):71-81.

34. Metzger R, Leichman CG, Danenberg KD, Danenberg PV, Lenz HJ, Hayashi K, et al. ERCC1 mRNA levels complement thymidylate synthase mRNA levels in predicting response and survival for gastric cancer patients receiving combination cisplatin and fluorouracil chemotherapy. J Clin Oncol 1998;16: 309-16.

35. Suganuma K, Kubota T, Saikawa Y, Abe S, Otani Y, Furukawa T, et al. Possible chemoresistance-related genes for gastric cancer detected by cDNA microarray. Cancer Sci 2003;94:355-9.

36. Fujita K, Kubota T, Matsusaki SW, Otani Y, Watanabe M, Teramoto T, et al. Further evidence for the value of the chemosensitivity test in deciding appropriate chemotherapy for advanced gastric cancer. Anticancer Res 1998;18:1973-8.

37. Abe S, Kubota T, Matsuzaki SW, Otani Y, Watanabe M, Teramoto $\mathrm{T}$, et al. Chemosensitivity test is useful in evaluating the appropriate adjuvant cancer chemotherapy for stage III nonscirrhous gastric cancer. Anticancer Res 1999;19:4581-86.

38. Kubota T, Egawa T, Otani Y, Furukawa T, Saikawa Y, Yoshida $\mathrm{M}$, et al. Cancer chemotherapy chemosensitivity testing is useful in evaluating the appropriate adjuvant cancer chemotherapy for stages III/IV gastric cancers without peritoneal dissemination. Anticancer Res 2003;23:583-8.

39. Samson DJ, Seidenfeld J, Ziegler K, Aronson N. Chemotherapy sensitivity and resistance assays: a systematic review. J Clin Oncol 2004:22:3618-30.

40. Schrag D, Garewal HS, Burstein HJ, Samson DJ, Von Hoff DD, Somerfield MR. American Society of Clinical Oncology Technology Assessment. Chemotherapy sensitivity and resistance assays. J Clin Oncol 2004;22:3631-8.

41. Weisenthal LM, Nygren P. Current status of cell culture drug resistance testing (CCDRT). 2002. http://weisenthal.org/ oncol_t.htm

42. Paik S, Shak S, Tang G, Kim C, Baker J, Cronin M, et al. A multigene assay to predict recurrence to tamoxifen-treated, nodenegative breast cancer. N Engl J Med 2004;351:2817-26.

43. Cappuzzo F, Varella-Garcia M, Shigematsu H, Domenichini I, Bartolini S, Ceresoli GL, et al. Increased HER2 gene copy number is associated with response to gefitinib therapy in epidermal growth factor receptor-positive non-small-cell lung cancer patients. J Clin Oncol 2005;23:5007-18.

44. Kubota T. Chemosensitivity test for adjuvant cancer chemotherapy of gastric cancer (JACCRO GC-04). Igaku no Ayumi (J Clin Exp Med) 2006;217.

45. Nomura E, Tanigawa N. JCOG clinical trial for evaluation of the utility of in vitro chemosensitivity test for cancer. Igaku no Ayumi (J Clin Exp Med) 2006;217. 